\title{
Layout of Sound Absorbing Materials in 3D Rooms Using Damping Contributions with Eigenvectors as Weight Coefficients"
}

\author{
Takao YAMAGUCHI $^{* *}$, Junichi TSUGAWA ${ }^{* * *}$, Hideki ENOMOTO ${ }^{* * * *}$ \\ and Yoshio KUROSAWA ${ }^{* * * *}$ \\ **Gunma University, \\ 1-5-1 Tenjin-cho, Kiryu, Gunma 376-8515, Japan \\ E-mail: yamagme3@gunma-u.ac.jp \\ ***Post Graduate School of Gunma University, \\ 1-5-1 Tenjin-cho, Kiryu, Gunma 376-8515, Japan \\ ****Fuji Heavy Industries Ltd., \\ 1-1 Subaru-cho, Ota, Gunma 373-8555, Japan
}

\begin{abstract}
In this study, we estimate an efficient layout of sound absorbing materials in three-dimensional spaces. For this purpose, we use damping contributions with eigenvectors as weight coefficients. Three-dimensional finite Elements of absorbing materials are modeled using a complex effective density and a complex bulk modulus of elasticity. By expanding the solution of complex eigenvalue problem with a small parameter related to damping, we derive the first order approximate equations of motion with respect to the small parameter. From these equations, the contribution of each element to modal damping is derived in consideration of multiple modes. The sound absorbing materials are arranged in three-dimensional spaces according to this contribution and by considering the components of eigenvectors at the evaluation points as the weight coefficients. The effectiveness of this method is verified using an expanded chamber.
\end{abstract}

Key words: Damping, Finite Element Method, Noise, Computer Aided Analysis, Modal Strain and Kinetic Energy Method

\section{Introduction}

Sound absorbing materials are frequently filled in three-dimensional spaces to suppress noise by means of dissipation of acoustic energy. For instance, expanded chambers filled with sound absorbing materials, are used in automotive mufflers. However, several experiments or numerical experiments are required for the determination of efficient layouts of the sound absorbing materials in spaces with complicated shapes like those of mufflers. Therefore, a method for systematically determining the layouts of these materials is desirable during design stages.

In several studies ${ }^{(1)}$ on experiments or theoretical/numerical analyses of sound absorption in rooms of architectural structures, a diffused sound field was assumed. These studies were generally concerned with the absorption of sound waves in intermediate/high frequency ranges in relatively large rooms. However, the sound fields in automobiles are sometimes not very large in comparison with the wavelength of the sound. Thus, in some cases, the sound fields in automobiles cannot be regarded as a diffused sound field. For such cases, in previous study ${ }^{(2) \sim(8)}$, the finite element method or the boundary element method, have been applied to sound absorption problems in sound fields with standing waves. These numerical methods are also applicable to analyze the sound fields that have arbitrary shapes. In these reports, calculation methods of frequency responses are mainly proposed. However,

Received 29 Sep., 2009 (No. T1-08-0357) Japanese Original : Trans. Jpn. Soc. Mech. Eng., Vol.74, No.747, C (2008), pp.2648-2654 (Received 21 Apr., 2008) [DOI: 10.1299/jsdd.4.166]

Copyright $\odot 2010$ by JSME 
sufficient information about both modal damping in the fields and the layout of the sound absorbing materials is not available. Yamaguchi ${ }^{(10)}$, one of the authors of this paper, previously modeled finite elements of sound absorbing materials by using complex effective density and complex bulk modulus of elasticity. Moreover, Yamaguchi (10) formulized the contributions of each element to modal damping in sound fields containing sound absorbing materials. The damping contribution of each element can be obtained by determining the approximate solutions to the complex eigenvalue problem. This approximate solution is determined using the finite element method for sound absorption. In this study, the solutions are expanded using a small parameter related to damping. Then, the damping contribution can be derived from the first order approximation with respect to the small parameter. Yamaguchi employed this damping contribution to develop a method for determining an efficient layout of sound absorbing materials in three-dimensional room for one spatial mode.

In this study, we extend our proposed method to determine the layout of sound absorbing materials for multiple spatial modes. We introduce the components of eigenvectors (i.e., acoustic modes) at the evaluation points as weight coefficients to investigate the layout. We use the weight coefficients to assess influences of the spatial modes at resonance on the frequency responses at the evaluation points. This method is applied to an expanded chamber that contains the sound absorbing materials. The dimensions of this chamber are determined by following Kagawa's experiment ${ }^{(2)}$. We compute the distributions of the damping contributions of each element in the chamber, and rearrange the materials according to the values of the multiplication product of the damping contribution and the weight coefficients. We verify the effectiveness of our proposed method using noise reduction between the initial layout and the rearranged layout.

\section{Numerical Method}

\subsection{Discrete equation of sound fields containing sound absorbing materials}

We discretize sound fields containing sound absorbing materials by using finite elements. In this study, the Helmholtz equation, which is suitable for a homogeneous sound field, is not used, because gas and the sound absorbing materials are mixed in a complicated manner. Thus, we adopt the following approach.

Under the assumption of infinitesimal amplitude, equations of motion of an inviscid compressive perfect fluid undergoing periodic oscillation can be expressed as follows ${ }^{(3)(9)(10)}$ :

$$
-\operatorname{grad} p=-\rho \omega^{2}\{U\}
$$

The continuity equation is expressed as follows:

$$
p=-E \operatorname{div}\{U\}
$$

where $p$ is the sound pressure, $\{U\}$ is the particle displacement vector, $\omega$ is the angular frequency, and $E$ and $\rho$ are the bulk modulus of elasticity and effective density of air, respectively.

By introducing shape functions $[N]^{T}$, the relationship between $p$ in an element and nodal sound pressure vector $\left\{p_{e}\right\}$ at the nodal points can be approximated.

$$
p=[N]^{T}\left\{p_{e}\right\}
$$

where $[N]^{T}=\left[N_{1}, N_{2}, N_{3, \ldots}\right]$, and $T$ denotes transpose.

Next, the kinetic energy, strain energy and external work are derived from Eqs. (1) (3). The following expressions are then obtained by applying the minimum energy principle.

$$
\begin{aligned}
& \left([K]_{e}-\omega^{2}[M]_{e}\right)\left\{p_{e}\right\}=-\omega^{2}\left\{u_{e}\right\} \\
& {[K]_{e}=\left(1 / \rho_{e}\right)[\widetilde{K}]_{e}}
\end{aligned}
$$




$$
[M]_{e}=\left(1 / E_{e}\right)[\tilde{M}]_{e}
$$

where $\rho_{e}$ and $E_{e}$ are the effective density and bulk modulus of elasticity for gas in an element, respectively. $\left\{u_{e}\right\}$ is the nodal particle displacement vector. $[\tilde{M}]_{e}$ is a matrix that contains the shape functions, whereas $[\widetilde{K}]_{e}$ is a matrix that contains the derivatives of the shape function. $\widetilde{M}_{e i j}$ and $\widetilde{K}_{e i j}$ are components of $[\tilde{M}]_{e}$ and $[\widetilde{K}]_{e}$, respectively. These components can be expressed as follows:

$$
\begin{aligned}
\tilde{M}_{e i j} & =\iiint_{e} N_{i} N_{j} d x d y d z \\
\widetilde{K}_{e i j} & =\iiint_{e}\left\{\left(\partial N_{i} / \partial x\right)\left(\partial N_{j} / \partial x\right)+\left(\partial N_{i} / \partial y\right)\left(\partial N_{j} / \partial y\right)+\left(\partial N_{i} / \partial z\right)\left(\partial N_{j} / \partial z\right)\right\} d x d y d z
\end{aligned}
$$

where $i$ is the component of the $i$ th row, and $j$ represents the component of the $j$ th column. In this paper, we refer to $[K]_{e}$ as the element stiffness matrix and $[M]_{e}$ as the element mass matrix.

A model employing complex effective density $\rho_{e}{ }^{*}$ and complex propagation speed $c_{e}{ }^{*}$ is used to analyze the sound fields inside the sound absorbing materials ${ }^{(6),(12)}$. In this paper, we use the following model having complex effective density $\rho_{e}{ }^{*}$ and complex bulk modulus of elasticity $E_{e}{ }^{*}=\rho_{e}{ }^{*}\left(c_{e}{ }^{*}\right)^{2}$ for the elements in the sound absorbing materials ${ }^{(9),(11)}$.

$$
\begin{gathered}
\rho_{e} \Rightarrow \rho_{e}^{*}=\rho_{e R}+j \rho_{e I} \\
E_{e} \Rightarrow E_{e}^{*}=E_{e R}+j E_{e I}
\end{gathered}
$$

Where $j$ is an imaginary unit. $E_{e R}$ and $E_{e I}$ are the real and imaginary parts of $E_{e}^{*}$, respectively. $E_{e I}$ is related to hysteresis between sound pressure $p$ and volume strain $\operatorname{div}\{U\} . \rho_{e R}$ and $\rho_{e I}$ are the real and imaginary parts of $\rho_{e}^{*}$, respectively. $\rho_{e I}$ is related to flow resistance $R$. We have verified effectiveness of this model in our previous study ${ }^{(9)}$.

The element mass matrix $[K]_{e}$ is obtained by substituting Eq. (9) into Eq. (5).

$$
[K]_{e}=\left[K_{R}\right]_{e}\left(1+j \eta_{e}\right)
$$

where

$$
\begin{aligned}
& {\left[K_{R}\right]_{e}=\left(\rho_{e R} /\left(\rho_{e R}^{2}+\rho_{e I}^{2}\right)\right)[\tilde{K}]_{e},} \\
& \eta_{e}=-\rho_{e I} / \rho_{e R}
\end{aligned}
$$

$\left[K_{R}\right]_{e}$ is the real part of the element stiffness matrix $[K]_{e}$. The imaginary part $\rho_{e I}$ of the effective density is related to flow resistance of the sound absorbing materials, Hence $\eta_{e}=-\rho_{e I} / \rho_{e R}$ corresponds to material damping due to the flow resistance.

By substituting Eq. (10) into Eq. (6), the element stiffness matrix $[M]_{e}$ is obtained.

$$
[M]_{e}=\left[M_{R}\right]_{e}\left(1+j \chi_{e}\right)
$$

where

$$
\begin{aligned}
& {\left[M_{R}\right]_{e}=\left(E_{e R} /\left(E_{e R}^{2}+E_{e I}^{2}\right)\right)[\tilde{M}]_{e},} \\
& \chi_{e}=-E_{e I} / E_{e R}
\end{aligned}
$$

$\left[M_{R}\right]_{e}$ is the real part of $[M]_{e} \cdot \chi_{e}$ is the damping effect due to hysteresis between pressure and volume strain in the sound absorbing materials.

All elements for the mixed sound field can be superposed using Eqs. (4)-(14). This 
results in the following discrete equation of a global system.

$$
\sum_{e=1}^{e_{\max }}\left(\left[K_{R}\right]_{e}\left(1+j \eta_{e}\right)-\omega^{2}\left[M_{R}\right]_{e}\left(1+j \chi_{e}\right)\right)\left\{p_{e}\right\}=-\omega^{2}\{u\}
$$

The size of the matrix and vectors in Eq. (15) is modified to be concurrent with the degree of freedom of the global system. $\{u\}$ is the nodal particle displacement vector. Both the stiffness matrix and the mass matrix for the fields containing gas and sound absorbing materials have complex parameters. Equation (15) corresponds to simultaneous equations having complex parameters. If known values are assigned to the excitation angular frequency $\omega$ and the nodal particle displacement vector $\{u\}$, Eq. (15) can be solved to obtain unknown $p$ for the frequency responses.

\subsection{Calculation of contribution of each element to modal damping}

We now present a calculation method to obtain the contributions of each element to modal damping for the mixed sound fields containing gas and sound absorbing materials.

Considering conditions of resonance, a homogeneous equation of Eq. (15) corresponds to the following complex eigenvalue problem:

$$
\sum_{e=1}^{e_{\max }}\left(\left[K_{R}\right]_{e}\left(1+j \eta_{e}\right)-\left(\omega^{(n)}\right)^{2}\left(1+j \eta_{t o t}^{(n)}\right)\left[M_{R}\right]_{e}\left(1+j \chi_{e}\right)\right)\left\{\phi^{(n)^{*}}\right\}=\{0\}
$$

where $e_{\max }$ denotes the number of the elements. $\left\{\phi^{(n)^{*}}\right\}$ is the complex eigenvector and $\eta_{t o t}^{(n)}$ is the modal loss factor. The superscript $(n)$ denotes the $n$th eigenmode. $\left(\omega^{(n)}\right)^{2}$ is the real part of the $n$th complex eigenvalue. Further, $\omega^{(n)}$ is the $n$th angular resonant frequency.

Next, the parameters $\beta_{s e}$ and $\beta_{k e}$ are introduced:

$$
\beta_{k e}=\eta_{e} / \eta_{\max }, \beta_{k e} \leq 1, \beta_{s e}=\chi_{e} / \eta_{\max }, \beta_{s e} \leq 1
$$

where $\eta_{\max }$ is the maximum value among the elements' material loss factors $\eta_{e}$ and $\chi_{e},\left(e=1,2,3, \ldots e_{\max }\right)$. Under the assumption of $\eta_{\max }<<1$, solutions of Eq.(16) are expanded using a small parameter $\mu=j \eta_{\max }$ :

$$
\begin{aligned}
& \left\{\phi^{(n)^{*}}\right\}=\left\{\phi^{(n)}\right\}_{0}+\mu\left\{\phi^{(n)}\right\}_{1}+\mu^{2}\left\{\phi^{(n)}\right\}_{2}+, \ldots \\
& \left(\omega^{(n)}\right)^{2}=\left(\omega_{0}^{(n)}\right)^{2}+\mu^{2}\left(\omega_{2}^{(n)}\right)^{2}+\mu^{4}\left(\omega_{4}^{(n)}\right)^{2}+, \ldots \\
& j \eta_{\text {tot }}^{(n)}=\mu \eta_{1}^{(n)}+\mu^{3} \eta_{3}^{(n)}+\mu^{5} \eta_{5}^{(n)}+, \ldots
\end{aligned}
$$

In these equations, under conditions of $\beta_{k e} \leq 1, \beta_{s e} \leq 1$ and $\eta_{\max }<<1$, we can obtain $\eta_{\max } \beta_{k e}<<1$ and $\eta_{\max } \beta_{s e}<<1$. Thus, both $\mu \beta_{s e}$ and $\mu \beta_{k e}$ are considered to be small parameters like $\mu$. In these equations, $\left\{\phi^{(n)}\right\}_{0},\left\{\phi^{(n)}\right\}_{1},\left\{\phi^{(n)}\right\}_{2}, \ldots$, $\left(\omega_{0}^{(n)}\right)^{2},\left(\omega_{2}^{(n)}\right)^{2},\left(\omega_{4}^{(n)}\right)^{2}, \ldots$ and $\eta_{1}^{(n)}, \eta_{3}^{(n)}, \eta_{5}^{(n)}, \ldots$ have real quantities.

The following equations are derived by substituting Eqs. (18), (19) and (20) into Eq. (16), and adopting the approximation from the $\mu^{0}$ order to the $\mu^{1}$ order:

$$
\begin{aligned}
& \eta_{t o t}^{(n)}=\eta_{k e}^{(n)}-\eta_{s e}^{(n)} \\
& \eta_{k e}^{(n)}=\sum_{e=1}^{e_{\max }}\left(\eta_{e} S_{k e}^{(n)}\right), \eta_{s e}^{(n)}=\sum_{e=1}^{e_{\max }}\left(\chi_{e} S_{s e}^{(n)}\right),
\end{aligned}
$$




$$
S_{k e}^{(n)}=\frac{\left\{\phi^{(n)}\right\}^{\mathrm{T}}\left[K_{R}\right]_{e}\left\{\phi^{(n)}\right\}}{\sum_{e=1}^{e_{\max }}\left\{\phi^{(n)}\right\}^{\mathrm{T}}\left[K_{R}\right]_{e}\left\{\phi^{(n)}\right\}}, \quad S_{s e}^{(n)}=\frac{\left\{\phi^{(n)}\right\}^{\mathrm{T}}\left[M_{R}\right]_{e}\left\{\phi^{(n)}\right\}}{\sum_{e=1}^{e_{\max }}\left\{\phi^{(n)}\right\}^{\mathrm{T}}\left[M_{R}\right]_{e}\left\{\phi^{(n)}\right\}}
$$

From these equations, the modal loss factor $\eta_{\text {tot }}^{(n)}$ can be obtained using the material loss factors $\eta_{e}$ of each element $e$ concerning $\rho_{e}{ }^{*}$, share $S_{k e}^{(n)}$ of the kinetic energy of each element to the total kinetic energy, material loss factors $\chi_{e}$ of each element $e$ concerning $E_{e}{ }^{*}$ and share $S_{s e}^{(n)}$ of the strain energy of each element to the total strain energy. Eigenmodes $\left\{\phi^{(n)}\right\}_{0}$ in Eq. (21) are real, and can be obtained by solving the real eigenvalue problem that corresponds to the equation by eliminating all damping terms in Eq. (16). Damping contributions of the elements to modal loss factors $\eta_{t o t}^{(n)}$ of the $n$th spatial resonance can be derived from Eq. (21) as follows:

$$
C_{e}^{(n)}=\eta_{e} S_{k e}^{(n)}-\chi_{e} S_{s e}^{(n)}
$$

The damping contributions $C_{e}^{(n)}$ in this equation indicate the influence that the positions of the sound absorbing materials exert on modal damping.

\subsection{Procedures for investigation of layout of sound absorbing materials for multiple modes}

For each element $e$ of the sound absorbing material, we define the sum of the damping contribution $C_{e}^{\text {tot }}$ of all elements over the multiple spatial modes as follows:

$$
C_{e}^{t o t}=\sum_{n=1} C_{e}^{(n)} W^{(n)}
$$

where $W^{(n)}$ is the weight coefficient, and $C_{e}^{(n)}$ is the damping contribution, that is defined for the $n$th spatial mode. $C_{e}^{\text {tot }}$ is introduced to analyze the necessity of an individual spatial mode by using the weight coefficient $W^{(n)}$ for generating efficient layouts of the sound absorbing materials. The following expression is used to represent the weight coefficient $W^{(n)}$ in Eq. (23).

$$
W^{(n)} \equiv\left|\phi_{\text {in }}^{(n)} \phi_{\text {out }}^{(n)}\right|
$$

$W^{(n)}$ is determined by $\phi_{i n}^{(n)}$ and $\phi_{o u t}^{(n)} . \phi_{i n}^{(n)}$ is a component of the $n$th eigenvector $\left\{\phi^{(n)}\right\}_{0}$ at the evaluation point near the sound source. Similarly, $\phi_{\text {out }}^{(n)}$ is the component of $\left\{\phi^{(n)}\right\}_{0}$ at the evaluation point near the sound receiver. The sound pressure levels at these evaluation points are affected by the magnitudes of the spatial modes (i.e., eigenvectors) at the evaluation points when the $n$th resonance occurs. The weight coefficients in Eq. (24) can be used to position the sound absorbing materials in the sound field, in order to intensively damp the spatial modes that strongly affect the sound pressure levels at the evaluation points.

$C_{e}^{t o t}$ is used in the following procedure to determine an efficient layout of the sound absorbing material. All the elements in $C_{e}^{\text {tot }},\left(e=1,2,3, \ldots, e_{\max }\right)$ are ordered by their magnitudes. We follow this order of magnitudes of the elements in $C_{e}^{\text {tot }}$ and select 
elements as the sound absorbing materials in the sound field. This process is continued until the sum of the volume for the selected elements reaches up to a specified value that has been determined in advance as the maximum volume of the sound absorbing material.

\section{Numerical Results}

\subsection{A three-dimensional model using finite elements for an expanded chamber}

To verify our method for estimating the layout of the sound absorbing material on the basis of the damping contribution, an acoustic finite element model of an expanded chamber (Fig.1) is used. The dimensions of this model are essentially similar to those of the model used in Kagawa's experiment ${ }^{(2)}$. Thus, the end of the outlet pipe is closed. We mainly used linear tetrahedral elements for this model. The hexahedral elements and wedge elements shown in Fig.1 are combinations of multiple tetrahedral elements. As

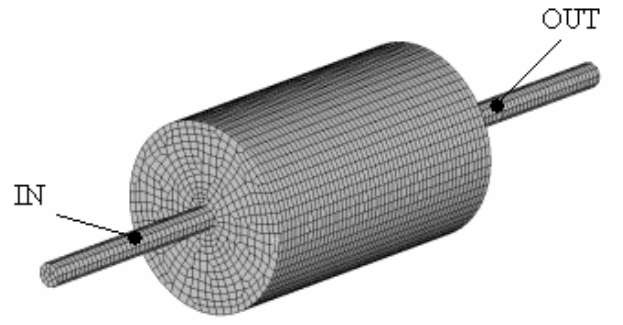

Fig.1 Acoustic finite element model of expanded chamber with sound absorbing material shown in Fig.1, the chamber has an expanded pipe, inlet pipe and outlet pipe. The expanded pipe has a large diameter of 170 $\mathrm{mm}$ and length of $272 \mathrm{~mm}$. Both the inlet pipe and the outlet pipe have small diameters of $21.5 \mathrm{~mm}$ and lengths of $200 \mathrm{~mm}$. "IN" in Fig. 1 denotes the evaluation point of sound pressure $p_{i n}$ at the inlet pipe, while "OUT" denotes the evaluation point of sound pressure $p_{\text {out }}$ at the outlet pipe. The distance between the evaluation points is $472 \mathrm{~mm}$. A point at the inlet pipe is excited by unit particle velocity $V$. The noise reduction level $\Delta p$ is calculated by using the following equation:

$$
\Delta p=20 \log _{10}\left|p_{\text {out }} / p_{\text {in }}\right|
$$

For this problem, $\phi_{i n}^{(n)}$ and $\phi_{\text {out }}^{(n)}$ for the weight coefficients $W^{(n)}$ in Eq.

correspond to the components of the $n$th eigenmodes (i.e.. eigenvectors) at the evaluation points at the inlet and outlet pipes, respectively. Larger influence on the sound pressure at the evaluation points result in the larger magnitudes in the components of the eigenvectors at the evaluation points. Thus, to absorb the sound pressure efficiently, we set larger weight coefficients $W^{(n)}$ for the modes, which have larger influences on the components of eigenvectors at the evaluation points.

\subsection{Accuracy of calculation of noise reduction level}

Firstly, the finite element model of the expanded chamber shown in Fig.1 is used to determine the accuracy of the calculated results of the noise reduction level $\Delta p$. To compute $\Delta p$, Eqs. (15) and (25) are used. As shown in Fig.2, the sound absorbing material is initially inserted in the expanded chamber by following Kagawa's experiment ${ }^{(2)}$. The painted elements in Fig.2 correspond to the sound absorbing material. The thickness of the material is $25 \mathrm{~mm}$. The sound absorbing material is arranged on the peripheral walls of the expanded part of the chamber. The sound absorbing material is also placed at the end of the outlet pipe. The volume of the sound absorbing material is $49.1 \%$ of the total volume of the expanded chamber. Hereafter, we call this model the "49.1\%-initial model" 
Figure 3 shows the calculated results of the noise reduction level $\Delta p$. In this figure, experimental results ${ }^{(2)}$ obtained by Kagawa are also written. In this figure, larger values of $\Delta p$ imply more noise reduction. As can be observed in this figure, the calculated and experimental results are in agreement with each other.

Next, the effects of the sound absorbing material on the noise reduction level $\Delta p$ are investigated as shown in Fig. 4. In Fig. 4, the solid line represents the calculated results when the sound absorbing material is filled in the chamber as shown in Fig.2, and the dotted line represents the calculated results when the material is removed from the chamber. For the dotted line (i.e., the results without the sound absorbing material), the troughs in the noise reduction level $\Delta p$ signify that the sound pressure level tends to be magnified by the acoustic resonance. In contrast, for the solid line (i.e., the results with sound absorbing material), the sharpness of the troughs decreases. Thus, the resonance is considerably damped due to the sound absorbing material. Figure 5 shows the related acoustic modes of the resonance in the cases without the sound absorbing material. The results from the fundamental to the 20th acoustic mode are shown in Fig.5. In this figure, the $n$th resonant frequencies are denoted as $f_{r}^{(n)}$, and the relevant weight coefficients $\quad W^{(n)}=\left|\phi_{\text {in }}^{(n)} \phi_{\text {out }}^{(n)}\right|$ are also displayed. The modes 2, 3, $4,5,8,9,14$ and 15 in Fig. 5 correspond to the troughs in the noise reduction without the sound absorbing material in Fig. 4. For these modes, the corresponding weight coefficients $W^{(n)}=\left|\phi_{\text {in }}^{(n)} \phi_{\text {out }}^{(n)}\right|$ are larger than those of the remaining modes. Therefore, we can expect to obtain the efficient layouts of the sound absorbing material by using these values of $W^{(n)}$, which can intensively damp the modes that

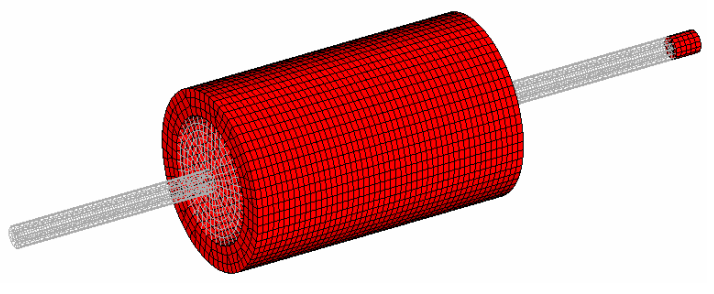

Fig. 2 49.1\%-initial model

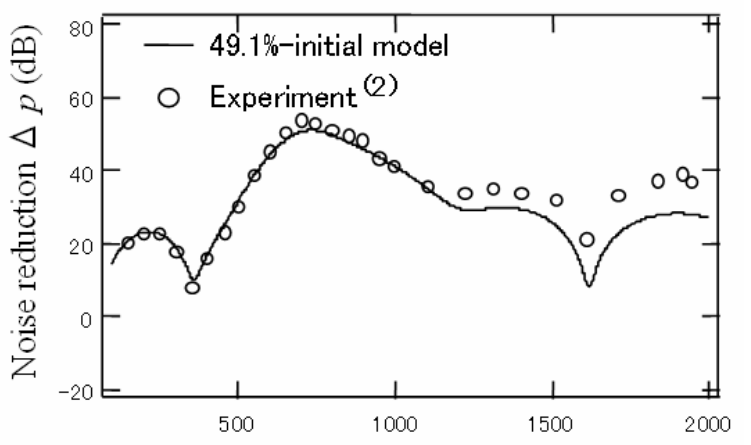

Frequency $(\mathrm{Hz})$

Fig. 3 Comparison between experimental results ${ }^{(2)}$ and calculated results

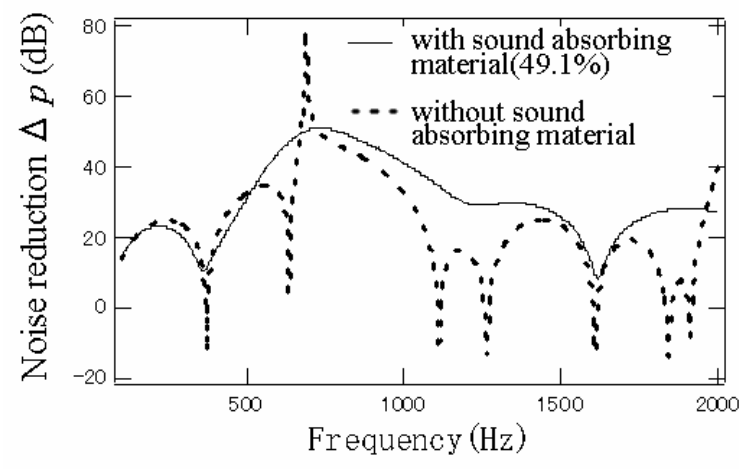

Fig. 4 Effect of sound absorbing material correspond to the troughs.

\subsection{Efficient layouts of sound absorbing material for multiple acoustic modes}

Next, we reposition the sound absorbing material from the " $49.1 \%$-initial model" in Fig. 2. 


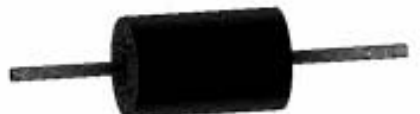

mode $1 f^{(4)}=0 \mathrm{~Hz},\left|\phi^{(n)} \phi^{(n)}\right|=2.22 \times 10$

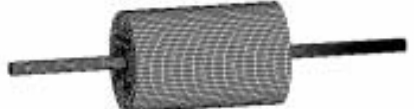

mode $2 f_{r}^{(2)}=370 \mathrm{~Hz},\left|\phi^{(n)} \phi^{(n)}\right|=1.65 \times 10$

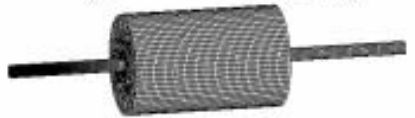

mode $3 f^{(3)}=415 \mathrm{~Hz},\left|\phi^{(n)} \phi^{(n)}\right|=1.47 \times 10^{\circ}$

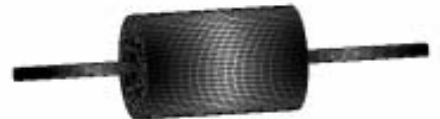

mode $4 f_{r}^{(4)}=635 \mathrm{~Hz},\left|\phi^{(*)} \phi(\infty)\right|=3.07$

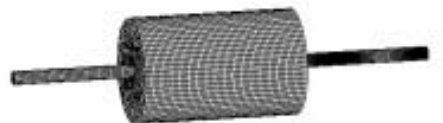

mode $5 f^{(5)}=1110 \mathrm{~Hz},\left|\phi^{(1)} \phi^{(n)}\right|=5.59 \times 10$

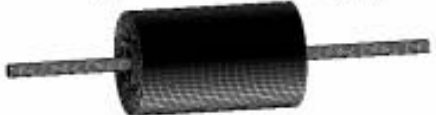

mode $6 f^{(0)}=1190 \mathrm{~Hz},\left|\phi^{(n)} \phi^{(0)}\right|=1.80 \times 10^{-4}$

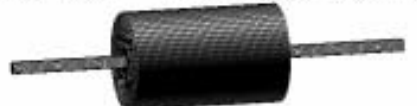

mode $T f_{r}^{(T)}=1190 \mathrm{~Hz},\left|\phi^{(n)} \phi^{(n)}\right|=3.38 \times 10^{-4}$

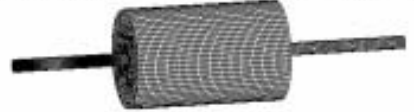

mode $8 f^{(1)}=1230 \mathrm{~Hz},\left|\phi_{w}^{(N)} \phi^{(\infty)}\right|=2.48 \times 10$

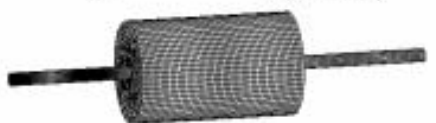

mode $9 f^{(1)}=1290 \mathrm{~Hz},\left|\phi^{(n)} \phi^{(n)}\right|=2.04 \times 10^{2}$

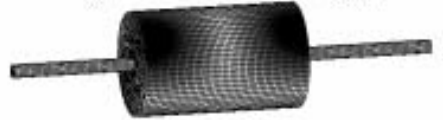

$\operatorname{mode} 10 f^{(10)}=1350 \mathrm{~Hz},\left|\phi^{(\cdots)} \phi^{(\cdots)}\right|=2.08 \times 10^{-1}$

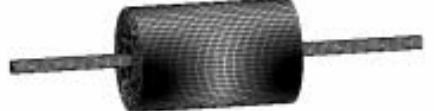

mode1 $1 f^{(114}=1350 \mathrm{~Hz},\left|\phi^{(n)} \phi^{(n)}\right|=2.12 \times 10^{-4}$

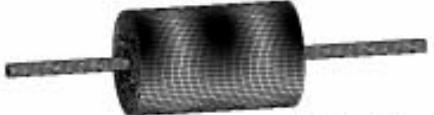

$\operatorname{mode1} f^{(12)}=1740 \mathrm{~Hz},\left|\phi^{(n)} \phi^{(\cdots)}\right|=6.22 \times 10^{-1}$

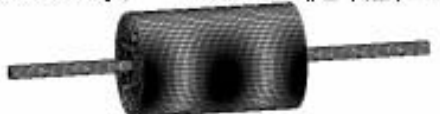

mode13 $f^{(1)}=1740 \mathrm{~Hz},\left|\phi^{(n)} \phi^{(n)}\right|=1.24 \times 10^{-4}$

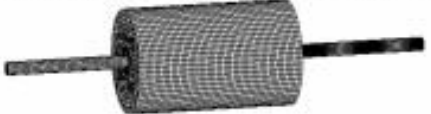

$\operatorname{mode1} 4 f^{(1+1)}=1840 \mathrm{~Hz},\left|\phi^{(n)} \phi^{(n)}\right|=9.28 \times 10$

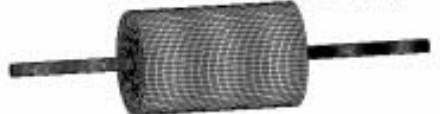

mode15 $f^{(1.5)}=1900 \mathrm{~Hz},\left|\phi^{(n)} \phi^{(n)}\right|=8.25 \times 10$

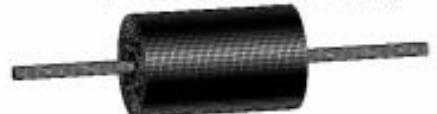

mode1 $6 f^{(n+)}=1970 \mathrm{~Hz},\left|\phi^{(n)} \phi^{(n)}\right|=1.60 \times 10^{-1}$

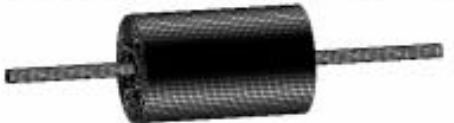

$\operatorname{mode1} f_{r}^{(17)}=1970 \mathrm{~Hz},\left|\phi^{(n)} \phi^{(n)}\right|=1.33 \times 10^{-1}$

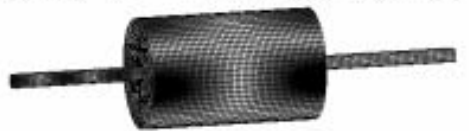

mode18 $f_{r}^{(1 x)}=2070 \mathrm{~Hz},\left|\phi^{(n)} \phi^{(n)}\right|=1.54$

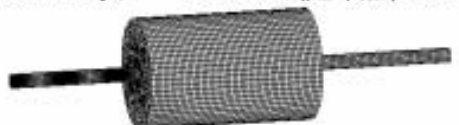

mode19 $f^{(10)}=2080 \mathrm{~Hz},\left|\phi^{(n)} \phi^{(n)}\right|=1.09 \times 10^{\circ}$

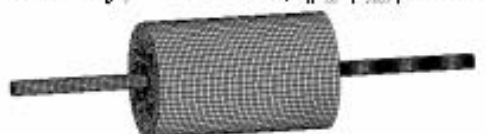

mode20 $f^{(20)}=2440 \mathrm{~Hz},\left|\phi^{(n)} \phi^{(n)}\right|=8.83 \times 10$

Fig. 5 Acoustic eigenmodes $\left\{\phi^{(n)}\right\}_{0}$ (distribution of sound pressure)

First, we compute the damping contributions $C_{e}^{(n)},\left(e=1,2,3, \ldots, e_{\max }\right.$ and $n=1,2$, $3, \ldots, 20)$ for the twenty acoustic modes shown in Fig. 5 using Eq. (22). Next, we use the weight coefficients $W^{(n)}$ to calculate the sum $C_{e}^{\text {tot }},\left(e=1,2,3, \ldots, e_{\max }\right)$ of the damping contributions as defined in Eq.(23). We then select the elements ordered by the magnitude of $C_{e}^{\text {tot }}$ as the sound absorbing material. This process is continued until the sum of the volumes of the selected elements reaches up to the specified volume of the sound absorbing material.

Figure 6 shows the repositioned model. This model is generated using the constant weight coefficients $W^{(n)}=1$. The specified volume is set as the same volume as that of the " $49.1 \%$-initial model". Hereafter, we call this model as the " $49.1 \%$ - improved model 
without weight coefficients". Figure 7 shows a comparison between the calculated noise reduction level $\Delta p$ of the "49.1\%- improved model without weight coefficients" shown in Fig.6 and that of the "49.1\%-initial model" shown in Fig.2. According to Fig. 7, $\Delta p$ for the repositioned model using

the constant $W^{(n)}$, is higher than $\Delta p$ for the initial model. Therefore, the sound absorption is enhanced using the sum of the damping contribution $C_{e}^{\text {tot }}$ under the constant weight coefficients $W^{(n)}=1$.

In the next analysis, we set $1.6 \%$ of the total volume of the chamber as the specified volume of the sound absorbing material. We introduce the weight coefficient as $W^{(n)}=\left|\phi_{\text {in }}^{(n)} \phi_{\text {out }}^{(n)}\right|$ to the related acoustic modes shown in Fig.5. Moreover, the sound absorbing material is repositioned by using $W^{(n)}=\left|\phi_{\text {in }}^{(n)} \phi_{\text {out }}^{(n)}\right|$ in Eq.(24).

Figure 8 shows the newly repositioned model. We call this model the "1.6\%-improved model with weight coefficients". In contrast, Fig.9 represents the repositioned model using the constant weight coefficients $W^{(n)}=1$. We call this model the " $1.6 \%$-improved model without weight coefficients". Figure 10 shows a comparison between the calculated noise reduction level $\Delta p$ of the "1.6\%-improved model with weight coefficients" and that of the "1.6\%-improved model without weight coefficients". As observed in Fig. 10, the noise reduction level increases with introduction of the weight coefficient as $W^{(n)}=\left|\phi_{\text {in }}^{(n)} \phi_{\text {out }}^{(n)}\right|$.

Therefore, the sound absorption is further enhanced due to the introduction of the weight coefficient using the components of the eigenmodes at the evaluation points.

\section{Conclusion}

In this study, we have proposed a method for determining an efficient layout of sound

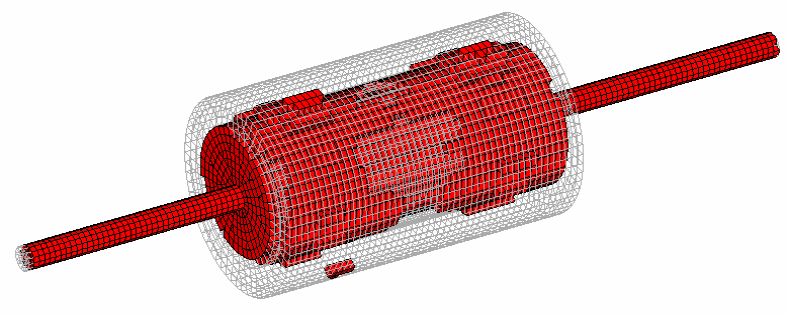

Fig.6 49.1\%-improved model without weight coefficients

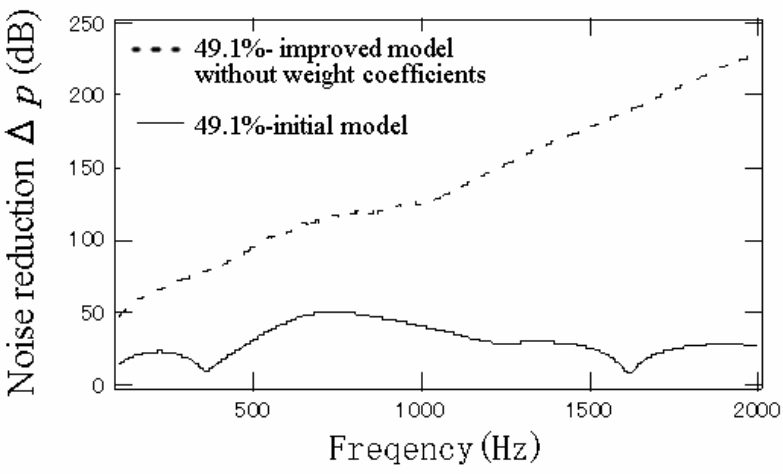

Fig.7 Comparison of $\Delta p$ between initial model and $49.1 \%$-improved model without weight coefficients

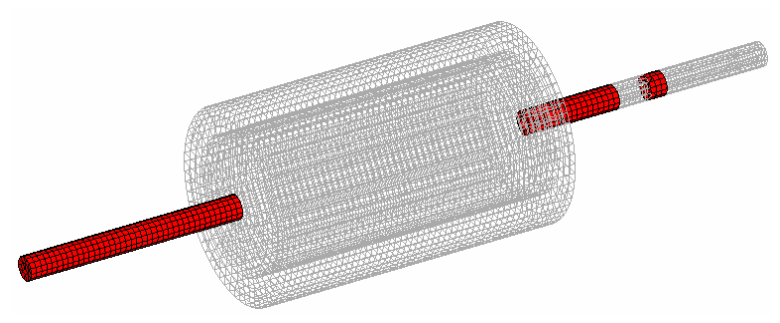

Fig. 8 1.6\%-improved model with weight coefficients

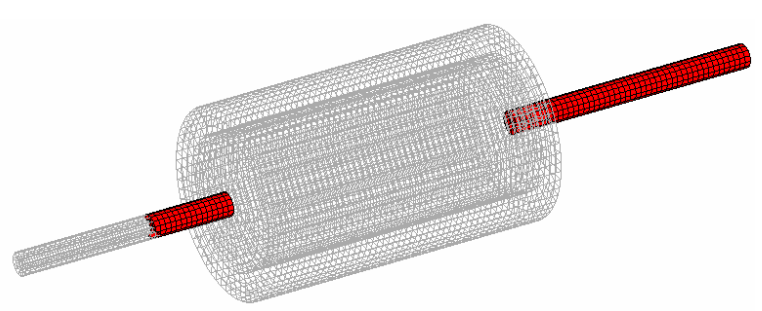

Fig. 9 1.6\%-improved model without weight coefficients 
absorbing materials in three-dimensional rooms using damping contributions with eigenmodes as weight coefficients. For finite elements of sound absorbing material, the complex effective density and the complex bulk modulus of elasticity are given. Discrete equations are formulated using the elements. Solutions to the complex eigenvalue problem of the discrete equations are expanded with a small parameter that is related to the damping parameter. The dominant equations of motion are derived by adopting the first order approximation with respect to the small parameter. The contribution of each element to modal damping can be obtained in consideration of multiple modes by arranging these derived equations. The sound absorbing materials are filled in the three-dimensional spaces according to these contributions. In this proposed method, the components of eigenvectors at the evaluation points are used as the weight coefficients. The weight coefficients play an important roll in intensively damping the acoustic modes

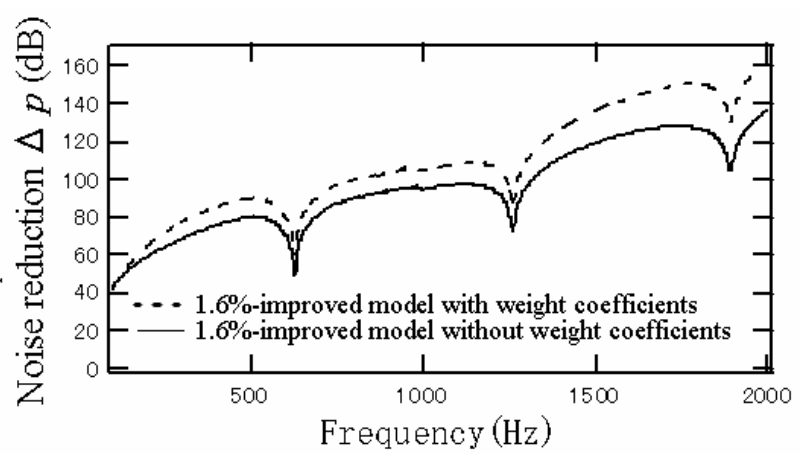

Fig. 10 Comparison of $\Delta p$ between 1.6\%improved models with and without weight coefficients that exert dominant influences on the sound pressure at the evaluation points. The effectiveness of this layout method has been verified using an expanded chamber.

\section{References}

(1)Zwikker, C. and Kosten, C., A., Sound Absorbing Materials, (1949), Elsevier Press.

(2)Kagawa, Y.,Yamabuchi, T. and Mori, A., Finite Element Simulation of an Axisymmetric Acoustic Transmission System with a Sound Absorbing Wall, Journal of Sound and Vibration, Vol.53, No.3 (1977), pp.357-374.

(3)Ejima, K., Ishii, T. and Murai, S., The Modal Analysis on the Acoustic Field, Journal of the Acoustical Society of Japan, Vol.44, No.6 (1988), pp.460-468.

(4)Craggs, A., A Finite Element Model for Rigid Porous Absorbing Materials, Journal of Sound and Vibration, Vol. 61, No.1 (1978), pp.101-111.

(5)Utsuno, H., Tanaka, T., Morisawa, Y. and Yoshimura, T., Prediction of Normal Sound Absorption Coefficient for Multi Layer Sound Absorbing Materials by using the Boundary Element method, Transactions of Japan Society of Mechanical Engineers, Vol.56, No.532C (1990), pp.3248-3252.

(6)Utsuno, H., Wu. T. W., Seybert, A. F. and Tanaka, T., Prediction of Sound Fields in Cavities with Sound Absorbing Materials, AIAA Journal, Vol.28, No.11 (1990), pp. 1870-1875.

(7)Kang, Y.J. and Bolton, S., Finite Element Modeling of Isotropic Elastic Porous Materials Coupled with Acoustical Finite Elements, Journal of the Acoustical Society of America, Vol.98, No.1 (1995), pp.635-643.

(8)Attala, N., Panneton, R., and Debergue, P., A Mixed Pressure-displacement Formulation for Poroelastic Materials, Journal of the Acoustical Society of America, Vol.104, No.3 (1998), pp.1444-1452.

(9)Yamaguchi, T., Approximated Calculation to Damping Properties of a Closed Sound Field Involving Porous Materials (Proposal of a Fast calculation Procedure for Modal Damping and Damped Response), Transactions of Japan Society of Mechanical Engineers, Vol.66, No.648C (2000), pp. 2563- 2569. 
(10)Yamaguchi, T., Kurosawa, Y. and Matsumura, S., Damped Analysis of 3D Acoustic Fields Involving Sound Absorbing Materials using FEM, Transactions of Japan Society of Mechanical Engineers, Vol.66, No.646C (2000), pp.1842-1848.

(11)Sato, S., Fujimori, T. and Miura, H., Sound Absorbing Wedge Design Using Flow Resistance of Glass Wool, Journal of the Acoustical Society of Japan, Vol.33, No.11 (1979), pp.628-636.

(12)Utsuno, H., Tanaka, T. and Fujikawa, T., Transfer Function Method for Measuring Characteristic Impedance and Propagation Constant of Porous Materials, Journal of the Acoustical Society of America, Vol.86, No.2 (1989), pp.637-643.

(13)Ma, B. A. and He, J. F., A Finite Element Analysis of Viscoelastically Damped Sandwich Plates, Journal of Sound and Vibration, Vol.152, No.1 (1992), pp.107-123.

(14)Biot, M. A., Theory of Propagation of Elastic Waves in a Fluid-Saturated Porous Solid, Journal of the Acoustical Society of America, Vol.28, No.2 (1955), pp.168-178.

(15)Allard, J. F., Propagation of Sound in Porous Media, (1993), Elsevier Applied Science.

(16)Zienkierwicz, O. C. and Cheung, Y. K., The Finite Element Method in Structural and Continuum Mechanics, (1967), McGraw-Hill. 